\title{
¿Cómo se orienta la apropiación del género lesson plan mediante la provisión de comentarios escritos en la práctica pedagógica en un programa de formación de profesores de inglés en Chile? ${ }^{1}$
}

\author{
How Is the Appropriation of the Lesson Plan Genre Guided through \\ Provided Written Comments in the Pedagogical Practice in a Training \\ Program for English Teachers in Chile?
}

\author{
Como orientar a apropriação do gênero lesson plan mediante a \\ provisão de comentarios escritos na prática pedagogica num programa \\ de formação de professores de Inglês em Chile?
}

Marta Patricia Meza García² Mónica Tapia Ladino ${ }^{3}$

Resumen

El presente artículo propone caracterizar a través de un estudio de caso cómo son los comentarios escritos (CE) más utilizados por un profesor universitario para orientar la elaboración del género lesson plan (LP) producido por los estudiantes en un programa de pregrado en Pedagogía en Inglés en el marco de su práctica docente en una institución universitaria de la ciudad de Concepción, Chile. En total se analizaron 432 CE obtenidos de 40 textos elaborados por 4 grupos de estudiantes. Los resultados de la investigación mostraron que la mayoría de los CE, de acuerdo a su composición, son simples o breves. En lo concerniente a la función de los CE se observó en los primeros niveles una mayor atención a los aspectos formales del lenguaje y en las fases finales una mayor atención al contenido de la lengua en los LP. Asimismo, se advierte que predomina la retroalimentación correctiva indirecta con especial atención sobre los aspectos gramaticales de la lengua. Los comentarios se presentan mayormente de un modo correctivo e imperativo.

Palabras clave: Retroalimentación, planificación de clases, comentarios escritos, segunda lengua

Abstract

This article proposes to characterize, through a case study, how are most often written comments used by a university teacher to guide the elaboration of the Lesson Plan (LP) genre produced by undergraduate students in a Teaching English program, as part of their teaching practice in a university in the city of Concepción, Chile. In total, we analyzed 432 written comments obtained from 40 texts elaborated by 4 groups of students. The research results showed that most of the writing comments are simple and brief according to their composition. Concerning their function, it was observed that in the early levels written comments focused mainly in formal aspects of the language, while in the final stages greater attention was paid to language content of the Lesson Plans. In the same way, a predominance of indirect corrective feedback is noticed with particular attention to the semantic aspects of the language. Comments are mostly presented in a corrective and imperative mode.

Keywords: Feedback, lesson plan, written comments, second language

1 El artículo es un trabajo investigativo llevado adelante por el equipo del proyecto Fondecyt 1140360 denominado: Los comentarios escritos y su relación con la elaboración de las tesis de pregrado en carreras de pedagogía de la ciudad de Concepción: análisis retórico discursivo de la construcción de un género académico a partir de su género primario.

2 Magister en Lingüística Aplicada, Universidad Católica de La Santísima Concepción, Chile. Correo electrónico: martapatriciamezagarcia3@gmail.com.

3 Departamento de Lenguas, Facultad de Educación, Vicerrectoría Académica, Universidad Católica de La Santísima Concepción. Chile. Correo electrónico: mtapia@ucsc.cl. 
Este artigo propõe caracterizar através de um estudo de caso como são os comentários escritos (cE) mais utilizados por um professor universitário para orientar o desenvolvimento Lesson Plan de gênero (LP), produzido pelos alunos em um programa de graduação em Ensino de Inglês como parte de sua prática de ensino em uma universidade na cidade de Concepcion, Chile. Em total foram analisados 432 CE obtidos de 40 textos produzidos por 4 grupos de alunos. Os resultados da pesquisa mostraram que a maioria dos CE, de acordo com a sua composição, são simples e curtos. Quanto ao papel do CE foi observado, nos primeiros níveis, uma maior atenção aos aspectos formais da linguagem, e nas fases finais uma maior atenção ao conteúdo do idioma no LP. Também se adverte o domínio do retroalimentação corretiva indireta com especial atenção sobre os aspectos gramaticais da língua. A maioria dos comentarios se apresentam de um modo correctivo e imperativo.

Palavras Chave: Retroalimentação, planificação de aulas, comentarios escritos, segunda língua

Artículo recibido el 19 de agosto de 2015 y aprobado el 27 de enero de 2016

\section{Introducción}

Una de las preocupaciones más prominentes en los últimos años en Chile es la calidad de la educación que se ofrece en el sistema escolar. Sin duda, son muchos los factores que se reúnen para obtener como resultado una educación de calidad. Uno de los aspectos determinantes se relaciona con la manera como las universidades y las entidades preocupadas de formar profesores enfrentan dicha tarea. En este sentido, la presente investigación aborda un aspecto de la formación de profesores de inglés orientado a la planificación de las actividades del aula. Las actividades de práctica que los estudiantes desarrollan durante su proceso formativo son determinantes para que lleguen a ser buenos profesores. En ellas, los estudiantes preparan materiales de clase que van utilizando de manera progresiva en los entornos escolares reales. Por lo tanto, la guía que reciben desde la universidad es determinante en su proceso formativo, pues busca asegurar un nivel de inglés estándar así como ajustar el escrito a las demandas profesionales. En este caso, nos interesa averiguar cómo se da el proceso de elaboración de los lesson plans, en adelante LP, durante las prácticas que se supervisan desde la universidad y cómo la retroalimentación desempeña un rol clave en la planificación de las actividades del aula escolar.

Las investigaciones sobre retroalimentación en relación con los procesos de formación en escritura académica en educación superior en Chile son relativamente nuevas, y este continúa siendo un campo poco explorado. En este contexto la provisión de retroalimentación por parte de los profesores a los textos escritos de sus estudiantes constituye una actividad recurrente; no obstante, el entrenamiento explícito para realizarla es escaso o nulo, razón por la cual constituye un objeto de estudio interesante. Una de las actividades más relevantes en la orientación de su desempeño profesional y en el aprendizaje de la segunda lengua para los futuros profesores de Pedagogía en Inglés es la elaboración de lesson plans durante su práctica pedagógica. Para lograr el dominio de dicha herramienta, la retroalimentación desempeña un rol central. Hyland y Hyland (2010) señalan la importancia de este proceso de retroalimentación escrita cuando afirman que toda la retroalimentación que los estudiantes reciben durante el proceso de composición y reescritura necesariamente tendrá un efecto positivo en su desempeño y en su desarrollo como escritores. Dicha retroalimentación a los LP se provee de diversas formas; en nuestro caso, nos enfocaremos en el análisis de los comentarios escritos en adelante CE.

Aunque los estudios sobre retroalimentación en procesos de enseñanza-aprendizaje de inglés como segunda lengua tienen una larga tradición (Duijnhouwer 2010; Ellis, 2009; Ferris, 1997; Hattie \& Timperley, 2007; Hyland \& Hyland, 2010; Perpigan, 2003, entre otros), son escasas hasta ahora las investigaciones que se han preocupado por estudiar la retroalimentación en contextos orientados a habilitar a los escritores-estudiantes en el dominio de un género discursivo profesional. El LP es un recurso común en la enseñanza de inglés como segunda lengua, utilizado en establecimientos educativos bilingües y no bilingües. En este sentido, 
nuestro artículo tiene como objetivo caracterizar cómo son los CE provistos por un profesor universitario para orientar la elaboración de LP producidos por estudiantes de un programa en Pedagogía en Inglés en el marco de su práctica docente.

La producción de un texto profesional es un proceso sistemático que demanda atención y constante revisión. Ofrecer comentarios escritos a los textos de los estudiantes es una actividad habitual (Straub, 1997) a la que los docentes le dedican mucho tiempo (Haswell, 2006; Sommer, 1982), en consecuencia, se espera que los estudiantes se guíen por ellos. Específicamente, nos interesa saber cómo son los CE que ofrece un profesor universitario para orientar la apropiación de un género profesional como el LP, a qué aspectos del escrito alude, el tono con el que se comunica y el grado de control que se ejerce a través de ellos al escrito del estudiante. Asimismo, interesa examinar cómo varían los $\mathrm{CE}$ a medida que se avanza en la formación, qué aspectos del género LP son más valorados y qué niveles de la lengua son más abordados en dichos CE.

A continuación presentamos algunos de los soportes teóricos que respaldan nuestra investigación. Ello incluye el concepto de género discursivo, nociones básicas sobre LP, retroalimentación en enseñanza de lenguas y caracterización y criterios de clasificación de los CE.

\section{Género discursivo}

El lesson plan constituye un género discursivo, en la medida en que circula en una comunidad de hablantes, en nuestro caso, un programa de formación universitaria de Pedagogía en Inglés. Dicho recurso se utiliza en situaciones comunicativas académicas en un aula de clase. En relación con ello, Bajtin (1985) afirma que un género es el resultado de un conjunto de convenciones y acuerdos establecidos por un grupo de hablantes en particular, frente al uso de la lengua en determinadas situaciones comunicativas con un propósito específico. En virtud de los rasgos prototípicos que definen un género, el LP es un tipo de ellos, dado que se trata de un discurso profesional que circula en situaciones formativas específicas y que responde a las exigencias particulares de un acto comunicativo profesional. En este sentido, Bazerman et ál. (2009) señalan que el género está mediado entre una situación social y los textos que responden estratégicamente a las exigencias de esas situaciones. Desde esta perspectiva, el LP constituye un tipo de género utilizado por una comunidad específica que comparte propósitos comunicativos afines, que apuntan al desarrollo y la aplicación de contenidos de tipo académico en una situación simulada de clases en el contexto del aula.

En nuestro estudio, se adopta el concepto de género discursivo desde la nueva retórica, perspectiva que reconoce la importancia del contexto y la naturaleza social de los géneros. Dicha aproximación busca averiguar cuáles son los objetivos comunicativos del discurso de la comunidad y cómo los rasgos generales de dicha comunidad ayudan a sus miembros a lograr sus objetivos sociales (Bawarshi \& Reiff, 2010). Según lo señalado, el LP es un género que transmite un conocimiento disciplinar específico con objetivos académicos y profesionales. En consecuencia, se hace necesario que los futuros profesores conozcan tales rasgos y principalmente sepan cómo utilizarlos en situaciones reales de enseñanza. De esta manera, y dado que el LP es un género típico del desempeño en el aula, los futuros profesores necesitarán utilizarlo en su ejercicio profesional. Esta visión considera los géneros como una organización textual discursiva y como acciones sociales complejas, dinámicas e interdependientes (Bazerman et ál., 2009, p. 6). Desde esta perspectiva, el LP es un género que se utiliza en situaciones profesionales simuladas de aprendizaje, para favorecer el proceso laboral pedagógico de los estudiantes en formación.

\section{Lesson Plans}

En el proceso formativo de los futuros profesores se considera la enseñanza de los géneros que utilizarán en el mundo profesional, y el LP es uno de ellos. En términos generales, un LP es un tipo de registro escrito de lo ocurrido en clase y que ayuda al profesor a recordar lo que ha decidido hacer durante la sesión (Harmer, 2001, p. 308). Al mismo 
Universidad Pedagógica Nacional

Facultad de Humanidades

tiempo, dicha herramienta contiene diferentes apartados. De hecho, Brown (2001) identifica cinco partes fundamentales en un lesson plan: revisión, introducción, presentación, práctica y evaluación. Atendiendo a las definiciones propuestas, un LP es una herramienta pedagógica mediante la cual los estudiantes practicantes o futuros profesores planifican los objetivos y contenidos que desarrollarán en un contexto real de clases. El LP considera además elementos como la distribución del tiempo de clase y las características específicas del grupo de escolares con quienes se implementará. Según Harmer (2001), la planificación de las clases es el arte de combinar una serie de elementos diferentes en un todo coherente para que la clase tenga una identidad y para que los estudiantes pueden reconocerla, trabajar dentro de ella y reaccionar ante cualquier metáfora que el profesor pueda utilizar para visualizar y crear esa identidad. En este sentido, la elaboración de LP durante el proceso de formación de los estudiantes de Pedagogía en Inglés constituye un elemento importante en la medida en que orienta a los futuros profesionales en la construcción y aplicación de herramientas pedagógicas adecuadas para el desarrollo de actividades en el aula. Por lo tanto, las directrices que recibe desde los académicos universitarios para ajustar tanto las actividades como los contenidos vertidos en el LP son determinantes para desarrollar clases de buena calidad, orientadas a obtener buenos resultados de aprendizaje en los estudiantes del sistema escolar.

\section{Retroalimentación en enseñanza de lenguas}

Una de las habilidades lingüísticas que representa mayor complejidad es la producción escrita. Al respecto, Ferris (2003) asegura que el aprendizaje de una segunda lengua es un proceso complejo en el que los estudiantes necesitan más asistencia para desarrollar sus habilidades de escritura. Debido a su complejidad, el aprendizaje de escritura en L2 requiere un trabajo bastante minucioso por parte del profesor universitario, que debe considerar la variedad de componentes que involucra y revisar constantemente los textos producidos por los estudiantes. En este contexto, la provisión de retroalimentación a través de CE es una herramienta esencial.

En términos generales, Duijnhouwer (2010) señala que existen relaciones significativas entre el proceso de escritura y el rendimiento, que puede mejorar mediante intervenciones que apunten a la planificación, la revisión y el seguimiento de los escritos. Por tanto, existe un vínculo relevante entre el proceso de escritura y la mejora que se puede lograr a través de la provisión de retroalimentación en forma de CE que los docentes ofrecen para que los estudiantes mejoren sus escritos. En el presente artículo, nos centramos en las teorías sobre retroalimentación en los procesos de enseñanzaaprendizaje $y$, en particular, en la que se provee en forma de comentarios escritos en procesos de aprendizaje de inglés como segunda lengua.

La retroalimentación se entiende como la información proporcionada por un agente externo con respecto a algunos aspectos de la ejecución de la tarea del alumno, destinados a modificar su cognición, y con el propósito de mejorar su rendimiento en algún área (Duijnhouwer, 2010). En este sentido, los CE que proveen los profesores constituyen un recurso para alentar a los estudiantes no solo a mejorar su desempeño en las tareas de escritura, sino también a consolidar su proceso de aprendizaje en esta habilidad. Visto desde esta perspectiva, la retroalimentación escrita entregada por el profesor en la elaboración de LP se orienta no solo a mejorar el texto escrito en su forma y contenido como resultado de una tarea específica, sino también a ajustar el escrito a los rasgos genéricos del LP en su contexto profesional.

\section{Los comentarios escritos}

Diferentes autores (Bitchener \& Ferris, 2012; Duijnhouwer, 2010; Ferris, 1997; Hyland, 2010 y Straub \& Lunsford, 1995) han estudiado la incidencia de la retroalimentación en los procesos de enseñanza-aprendizaje de inglés como primera y segunda lengua. Para efectos de este artículo, hemos definido un conjunto de propuestas taxonómicas que resultaron útiles para nuestros objetivos. 
En el marco de la enseñanza del inglés como primera lengua, Straub \& Lunsford (1995) proponen una manera de caracterizar las respuestas escritas que proveen académicos universitarios a los textos elaborados por estudiantes de primer año. De dicha clasificación, interesa para efectos de esta investigación, la categoría de modo, que se define como la expresión de control que se ejerce sobre el texto, ya sea como evaluación negativa, pregunta solución, mandato, felicitación, entre otros. También adoptamos la propuesta de Brown (2001) en lo que respecta a la ubicación de los CE. En este caso, a qué partes del lesson plan alude el CE. Se consideran cinco partes básicas que puede contener un LP: (1) los objetivos, entendidos como aquello que se quiere lograr a través de la aplicación del LP en la lección con los estudiantes; (2) la introducción es la parte inicial de la clase, en la cual se hace la presentación del nuevo tema; (3) la presentación, que constituye la parte del LP en la que se dan a conocer las nuevas estructuras o el nuevo vocabulario que se trabajará durante la sesión de clases; (4) la práctica, en la cual se indican las actividades que el estudiante ha de realizar con los nuevos conceptos o el vocabulario adquirido en la sesión de clase; y (5) la producción, sección en la que se indica lo que los estudiantes hacen mediante ejercicios u otras actividades, el vocabulario y las estructuras adquiridas durante la sesión a través de la implementación del LP.

Incorporamos, además, el criterio de foco propuesto por Hyland (2003), quien señala que el comentario se focaliza cuando el profesor escoge solo uno o dos aspectos del texto de sus estudiantes para corregirlos e indica explícitamente cuál es el problema. No es focalizado cuando se provee un comentario general, que impide identificar el problema de escritura.

Otra mirada en relación con la provisión de retroalimentación es la propuesta por Duijnhouwer (2010), quien propone una serie de criterios de los cuales adoptamos tres: estrategia, composición y objeto. La primera se define como la provisión de alguna herramienta o un plan de acción alternativo para encontrar soluciones de mejora a los problemas señalados en el texto, sin indicar una solución específica. En cuanto a la composición del CE (tipo), identifica comentarios simples y compuestos. Son simples aquellos que contienen sólo un juicio (positivo o negativo) y aluden a un solo objeto. Son compuestos aquellos CE que o bien contienen más de un juicio de calidad, o bien aluden a objetos diferentes. La tercera categoría es la de objeto, que se define como el aspecto del escrito al que alude el comentario, con independencia del apartado. Dentro de la categoría objeto escogimos sólo tres de los criterios propuestos por la autora: uso de la lengua, contenido y estructura. Se entiende por uso de la lengua aquellos aspectos léxicos, gramaticales, ortotipográficos y de puntuación a los que alude el comentario; el contenido tiene que ver con las ideas o aspectos semánticos del escrito, y la estructura hace referencia a la organización lógica de la información tanto a nivel intra como interpárrafos.

La revisión bibliográfica indica que la retroalimentación correctiva es la que goza de mayor tradición. Bitchener \& Ferris (2012) la definen como aquella que alude a los errores o fallas en el uso del lenguaje como código. Los autores distinguen dos subcategorías: directo e indirecto. El directo es aquel en el que el docente provee la forma correcta para el error señalado en el texto del estudiante, y el indirecto es aquel en el que solo señala o destaca el error, sin proveer la forma correcta para enmendarlo.

\section{Metodología}

La investigación cuantitativa se basó en un estudio de caso, con un diseño de tipo exploratorio secuencial (Hernández Sampieri et ál., 2008, p. 163). En nuestro estudio, no hubo manipulación de variables, sino que se definió una serie de categorías de análisis que se obtuvieron de dos tipos de fuentes de datos: las bibliográficas y el análisis de muestras preliminares. Con respecto a los criterios basados en la revisión bibliográfica se identificó un conjunto de criterios de análisis que comprenden ocho categorías, definidas a partir de la revisión de los estudios de Straub \& Lunsford (1995), Ferris (1997), Hyland \& Hyland (2010), Ellis (2009), Duijnhouwer (2010) y Bitchener \& Ferris ( 2012). 
Universidad Pedagógica Nacional

Facultad de Humanidades

\section{Recolección de datos}

Al inicio de la investigación se contactó a un docente informante y se solicitó su participación previo consentimiento informado. El profesor informante nos permitió acceder al material que había almacenado en su computador.

\section{Participantes}

Se trabajó con materiales provenientes de cuatro grupos de estudiantes de los semestres 2, 5, 6 y 7 del programa de pregrado en Pedagogía en Inglés que cursaban la asignatura de Práctica Docente en la Universidad Católica de la Santísima Concepción en Concepción, Chile. La muestra estuvo constituida por los CE provistos por un docente a cargo de los cursos de Práctica Pedagógica a los lesson plans escritos por 40 estudiantes, distribuidos en los 4 grupos. Los materiales fueron elaborados entre 2008 y 2013 en el marco del curso en el que se les enseñaba a elaborar los LP que luego serían implementados en las aulas del sistema escolar. En total, la muestra quedó constituida por 432 CE provistos a los LP de los 40 estudiantes.

\section{Instrumento}

Con el objetivo de caracterizar los CE provistos por el profesor para orientar la elaboración de LP producidos por sus estudiantes, se elaboraron e identificaron los criterios para la clasificación. Todos los criterios de análisis fueron elaborados para ser aplicados de manera independiente a cada CE. Esto quiere decir que un comentario puede ser analizado con cada uno de los criterios que se describen en la tabla 1. Las categorías de análisis se obtuvieron de dos fuentes de datos: las bibliográficas y el análisis de muestras preliminares. Se consideraron ocho categorías, a saber: número de palabras, composición del CE (simple o compuesto), sección, objeto, correctivo, estrategia, modo y foco.

En la tabla 1, presentamos la tabla detallada con los criterios de análisis de la retroalimentación producto de la clasificación. Además, se incorporaron ejemplos extraídos de los LP corregidos por el docente. En la primera columna se indican las categorías, luego las subcategorías, y finalmente los ejemplos correspondientes a cada una de ellas.

\section{Análisis de la información}

El análisis de los datos se realizó siguiendo un criterio inductivo. Se revisó cada CE y se caracterizó según cada uno de los criterios explicitados en la tabla anterior. Para efectuar dicho análisis, los datos se ingresaron en una tabla de Excel, en la cual se consignó información sobre el tipo de práctica pedagógica, los trozos de texto del estudiante aludido por el CE y el comentario que hizo el profesor. Los CE se clasificaron en primera instancia en simples y compuestos. Posteriormente, los compuestos se descompusieron en simples. Realizado el quiebre de los compuestos se procedió a clasificar cada uno de los CE aplicando los criterios de análisis de las categorías restantes. En las planillas se ingresaron los rasgos de los CE con criterios numéricos que los identificaban. Se hicieron cálculos de estadística simple de promedios mínimo y máximo, desviación estándar, media, distribución de la frecuencia, entre otros.

\section{Resultados}

Los resultados se han organizado en tres grupos: el primero corresponde a los criterios generales de los textos, entre los cuales destacamos: número de textos, número total de palabras por texto y promedio de palabras por texto; el segundo se relaciona con los aspectos generales de los CE, como su cantidad por texto, número total de palabras, promedio de palabras, extensión mínima y máxima, promedio por texto, y tasa de provisión por número de palabra del texto comentado; el tercero presenta las distribuciones de los CE según las categorías de análisis: composición, sección, objeto, correctivo, enfoque, estrategia y modo.

En la tabla 2 se presentan los rasgos generales de los CE en términos del número total de textos analizados, el número total de palabras por texto y el promedio de palabras por texto. 
Tabla 1. Categorías y subcategorías de análisis

\begin{tabular}{|c|c|c|}
\hline CATEGORIÁA & SUBCATEGORÍA & EJEMPLOS \\
\hline $\begin{array}{l}\text { 1. Número de } \\
\text { palabras } \\
\text { (Duijnhouwer, 2010) }\end{array}$ & & $\begin{array}{l}\text { 1. Always in capital letter ( } 4 \text { palabras) } \\
\text { 2. going to ( } 1 \text { palabra) }\end{array}$ \\
\hline $\begin{array}{l}\text { 2. Composición del CE } \\
\text { (Duijnhouwer, 2010) }\end{array}$ & $\begin{array}{l}\text { Simple } \\
\text { Compuesto }\end{array}$ & $\begin{array}{l}\text { He is in the bedroom } \\
\text { How does this activity introduce ss. to the topic "Telling the time"? / Is it } \\
\text { an engaging activity? / Is it possible for ss. to recall previous knowledge? }\end{array}$ \\
\hline \multirow{5}{*}{$\begin{array}{l}\text { 3. Sección } \\
\text { (Brown, 2001) }\end{array}$} & Objetivos & What's the purpose of asking this? \\
\hline & Introducción & How does this activity introduce ss. to the topic "Telling the time"? \\
\hline & Presentación & You need to think of a more natural and engaging activity \\
\hline & Práctica & You can name, give opinion or describe \\
\hline & Producción & What did we learn today? \\
\hline $\begin{array}{l}\text { 4. Objeto } \\
\text { (Duijnhouwer, 2010) }\end{array}$ & $\begin{array}{l}\text { Uso de la lengua } \\
\text { Contenido } \\
\text { Estructura }\end{array}$ & $\begin{array}{l}\text { "after listening" } \\
\text { "not clear" } \\
\text { "Better to stay an aim from Mineduc's proposal." }\end{array}$ \\
\hline \multirow{3}{*}{$\begin{array}{l}\text { 5. Correctivo } \\
\text { (Bitchener \& Ferris, 2012) }\end{array}$} & Directo & "Name and describe clothes" \\
\hline & Indirecto & "This is not coherent to aim" \\
\hline & No aplica & There is no "natural" context setting \\
\hline \multirow{2}{*}{$\begin{array}{l}\text { 6. Estrategia } \\
\text { (Duijnhouwer, 2010) }\end{array}$} & Con estrategia & $\begin{array}{l}\text { "Mime an action which represents what we do in that room. } \\
\text { For example: ..." }\end{array}$ \\
\hline & Sin estrategia & "This is not an effective way of checking understanding." \\
\hline \multirow{6}{*}{$\begin{array}{l}\text { 7. Modo } \\
\text { (Straub \& Lunsford, 1995) }\end{array}$} & Felicitación & "Very pertinent activity!" \\
\hline & Orden & Check question form \\
\hline & Consejo & These should be linguistic problems your ss. might face \\
\hline & Requerimiento indirecto & "Is this an engaging activity?" \\
\hline & Pregunta - problema & $\begin{array}{l}\text { How does this activity introduce ss. to the topic of "Telling the time"? Is it } \\
\text { an engaging activity? Is it possible for ss. to recall previous knowledge? Do } \\
\text { you think this is an engaging activity? }\end{array}$ \\
\hline & Evaluación negativa & Not clear instruction \\
\hline $\begin{array}{l}\text { 8. Foco } \\
\text { (Hyland, 2003) }\end{array}$ & $\begin{array}{l}\text { Focalizado } \\
\text { No focalizado }\end{array}$ & $\begin{array}{l}\text { shown-presented } \\
\text { Mising info }\end{array}$ \\
\hline
\end{tabular}

Tabla 2. Totales de textos, palabras por texto y promedio

\begin{tabular}{|c|c|c|c|}
\hline PRÁCTICA & N. ${ }^{\circ}$ DE LP & $\begin{array}{c}\mathbf{N}^{\circ} \text { TOTAL } \\
\text { PALABRAS } \\
\text { POR TEXTO }\end{array}$ & $\begin{array}{c}\text { PROMEDIO DE } \\
\text { PALABRAS } \\
\text { POR TEXTO }\end{array}$ \\
\hline 2 & 11 & 3290 & 299 \\
\hline 5 & 10 & 6148 & 558 \\
\hline 6 & 8 & 4215 & 526 \\
\hline 7 & 11 & 7382 & 671 \\
\hline Totales & 40 & 21035 & 525 \\
\hline
\end{tabular}

Como se muestra en la tabla 2, en total se analizaron 40 lesson plans elaborados por los estudiantes distribuidos en los 4 grupos que conformaban la muestra. Los 40 textos suman un total de 21035 palabras. Al observar los datos del número de palabras por textos, con relación al semestre, se advierte un aumento directamente proporcional en el número de palabras por semestre, es decir, a medida que se avanza en el semestre también aumenta el número de palabras producidas por los estudiantes en sus textos. Ese incremento podría significar que los estudiantes van logrando un mayor dominio del código de la segunda lengua, lo cual les permite escribir con más facilidad. Otra razón podría ser que la tarea se torna más compleja o que las exigencias con respecto a la producción escrita aumentan.

Presentamos a continuación la tabla 3, que contiene la información correspondiente al número de CE por texto, número total de palabras, promedio de palabras, valores mínimo y máximo por texto, cantidad promedio por texto y tasa por número de palabra del texto comentado. 
Universidad Pedagógica Nacional

Facultad de Humanidades

Tabla 3. Número de comentarios escritos y de palabras, y tasa de comentario escrito por texto y palabra

\begin{tabular}{|c|c|c|c|c|c|c|c|c|}
\hline $\begin{array}{c}\text { PRÁCTICA } \\
\mathrm{N}^{\circ}{ }^{\circ}\end{array}$ & N..$^{\circ}$ DE LP & $\begin{array}{l}\text { N. }{ }^{\circ} \text { DE } \\
\text { CE POR } \\
\text { TEXTO }\end{array}$ & $\begin{array}{c}\text { N. }{ }^{\circ} \text { TOTAL DE } \\
\text { PALABRAS } \\
\text { POR (CE) }\end{array}$ & $\begin{array}{l}\text { PROMEDIO } \\
\text { DE PALABRAS } \\
\text { POR CE }\end{array}$ & $\begin{array}{l}\text { MÍN. DE CE } \\
\text { POR TEXTO }\end{array}$ & $\begin{array}{l}\text { MÁX. DE CE } \\
\text { POR TEXTO }\end{array}$ & $\begin{array}{l}\text { PROMEDIO } \\
\text { DE CE POR } \\
\text { TEXTO }\end{array}$ & $\begin{array}{c}\text { TASA DE } \\
\text { CE POR } \\
\text { PALABRA }\end{array}$ \\
\hline 2 & 11 & 50 & 322 & 6 & 1 & 7 & 4 & 65 \\
\hline 5 & 10 & 62 & 396 & 6 & 2 & 11 & 6 & 99 \\
\hline 6 & 8 & 100 & 575 & 5 & 8 & 15 & 12 & 42 \\
\hline 7 & 11 & 220 & 889 & 4 & 1 & 39 & 20 & 33 \\
\hline Totales & 40 & 432 & 2182 & 5 & 1 & 39 & 11 & 48 \\
\hline
\end{tabular}

Como se observa en la tabla 3 , el número total de CE es de 432 distribuidos en los 40 LP elaborados por los alumnos. Dichos CE suman en total 2182 palabras. Los LP tenían como mínimo 1 CE y 39 como máximo en los grupos 2 y 7 , respectivamente. En promedio cada CE escrito contenía entre 4 y 6 palabras. Nuestros resultados son menores a los encontrados en el estudio realizado por Ferris (1997), quien señala que sus comentarios contenían un promedio de 6 a 15 palabras. En términos generales, cada LP es comentado con $11 \mathrm{CE}$ en promedio y la tasa de provisión del CE es cada 48 palabras. Nuestros datos, en relación con el promedio de CE por texto, coinciden con los hallazgos de Hyland y Hyland (2010), quienes identificaron una tasa de provisión de 9 a 14 CE por página. Asimismo, la tasa de provisión de CE en nuestra investigación es menor a la encontrada en el estudio de Tapia- Ladino, Correa y Arancibia (2014) en borradores de tesis en carreras de Pedagogía en la ciudad de Concepción. Las investigadoras encontraron que cada 102 palabras, en promedio el profesor de Español como lengua materna provee un comentario simple al borrador de estudiantes universitarios.

En el gráfico 1 se presenta la distribución general producto del análisis de la clasificación realizada y que caracteriza los CE provistos por el profesor en las categorías identificadas: tipo (simple o compuesto), sección, objeto, correctivo, enfoque, estrategia, y modo.

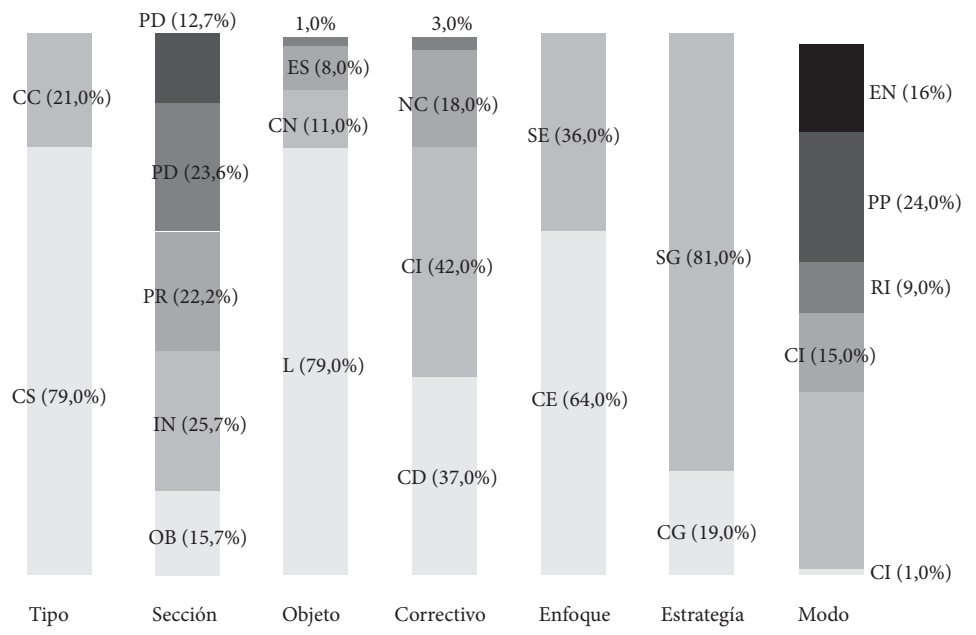

Gráfico 1. Distribución general de los CE según categorías y subcategoría de análisis

\footnotetext{
Tipo: cs comentario simple, cc comentario compuesto

Objeto: UL uso de la lengua, CN contenido, Es estructura

Enfoque: CE con enfoque, sE sin enfoque

Modo: FL felicitación, OD orden, CJ consejo, RI requerimiento indirecto, PP pregunta problema, EN evaluación negativa

Sección: ов objetivos, IN introducción, PR presentación, PC práctica, PD producción

Correctivo: CD correctivo directo, CI correctivo indirecto, NC no correctivo

Estrategia: CG con estrategia, SG sin estrategia
} 
Como se advierte en el gráfico 1 , los CE en su composición son predominantemente simples y de tipo correctivo indirecto. En un sentido, nuestros datos coinciden con los hallazgos de Ferris (1997), cuyos resultados indicaron - en lo relacionado con las diferencias entre los comentarios marginales y finales- que los comentarios marginales son normalmente preguntas cortas. Una de las conclusiones más relevantes del estudio realizado por Ferris (1997) es que los estudiantes prestan mucha atención a los comentarios y observaciones hechas por el profesor, lo cual les ayuda a tomar decisiones sobre el texto y a corregirlo. En nuestro caso, el análisis muestra que el profesor hace correcciones de tipo indirecto en las cuales entrega pistas o explicaciones, sin proveer la forma correcta en un $42 \%$ de los casos. Aproximadamente en un 37\% la retroalimentación del docente es correctiva directa, es decir, proporciona la corrección del error. Lo anterior concuerda con los resultados de Hyland y Hyland (2010), quienes en un estudio averiguaron si la retroalimentación a los errores ayuda a los escritores. Los investigadores concluyen que el $45 \%$ de la retroalimentación entregada es de tipo correctivo directo y el $40 \%$ es correctivo indirecto. El estudio señala que en la mayoría de los casos el estudiante es capaz de corregir exitosamente los errores marcados por el profesor. En nuestros datos, también se advierte que el $18 \%$ de la retroalimentación que suministra el docente es no correctiva, y el 3\% hace alusión a aspectos que no tienen que ver con el uso del código, a saber: organización de las temáticas y registro de la lengua.

Por otra parte, se evidencia que la mayor parte de los CE entregados por el docente (81\%) no provee estrategia y el $64 \%$ de los CE son focalizados (con enfoque). En relación con los resultados en la categoría Estrategia, nuestros datos difieren de los resultados obtenidos por Duijnhouwer (2010), quien en uno de sus estudios experimentales sobre retroalimentación y motivación encontró que los estudiantes mejoran la calidad de sus textos cuando reciben estrategias explícitas de mejoramiento para implementar en el texto. Esta modalidad promueve la escritura más autónoma. Con respecto a la cate- goría Foco, nuestros resultados son mayores a los señalados por Straub y Lunsford (1995), quienes en su estudio en el ámbito universitario analizaron los CE según los criterios de foco y modo. De acuerdo a lo señalado por los investigadores, el $21 \%$ de los comentarios centraron su foco exclusivamente en la estructura de las oraciones, la fraseología y errores generales.

En cuanto al modo en cómo se proveen los $\mathrm{CE}$, se advierte que el más empleado por el profesor es la orden $(33 \%)$, y el menos utilizado corresponde a la felicitación (1\%). En lo que respecta al objeto, el porcentaje más alto corresponde a uso de la lengua, con un 79\%. Tal resultado es consistente con los análisis de los CE según el criterio correctivo. Ello indica que el profesor valora el dominio de la lengua como código.

Finalmente, las secciones del LP con mayor tasa de provisión de CE son la introducción y la práctica con un $25 \%$ y un $23 \%$, respectivamente. El alto porcentaje en la introducción se podría deber a que implica para los estudiantes un mayor esfuerzo iniciar la producción de un texto o mantener su continuidad dado que es una parte en la que deben ser concretos. En lo concerniente a la sección de producción, esta es la menos comentada (12\%). Una posible causa para ello podría ser que por su ubicación al final del LP, la carga cognitiva de los estudiantes sea menor, o bien que aún presentan cierta resistencia frente al manejo del código de la segunda lengua. Así lo señalan Ferris (2006, 2010) en estudios realizados con el propósito de averiguar si la retroalimentación correctiva escrita ayuda a los estudiantes a mejorar cierto tipo de errores más que otros. Los autores concluyen que ciertos tipos de errores, sin duda, parecen ser más sensibles a la retroalimentación correctiva escrita. Sin embargo, hubo algunas variaciones individuales. Lamentablemente no fue posible establecer comparaciones estadísticas con relación a la categoría Sección para establecer las preferencias del profesor o las razones acerca de la distribución de CE asociados a cada parte del LP. 
En el gráfico 2 se presenta la distribución de los CE según la categoría Objeto, en la cual se consideraron sólo tres criterios: uso de la lengua, contenido y estructura (Straub \& Lunsford, 1995). Se excluyeron en nuestra clasificación criterios globales de los textos referidos a reflexiones sobre el escrito y las correcciones que aludían a los aspectos extratextuales, como intención o propósito de los escritores, tema tratado o audiencia.

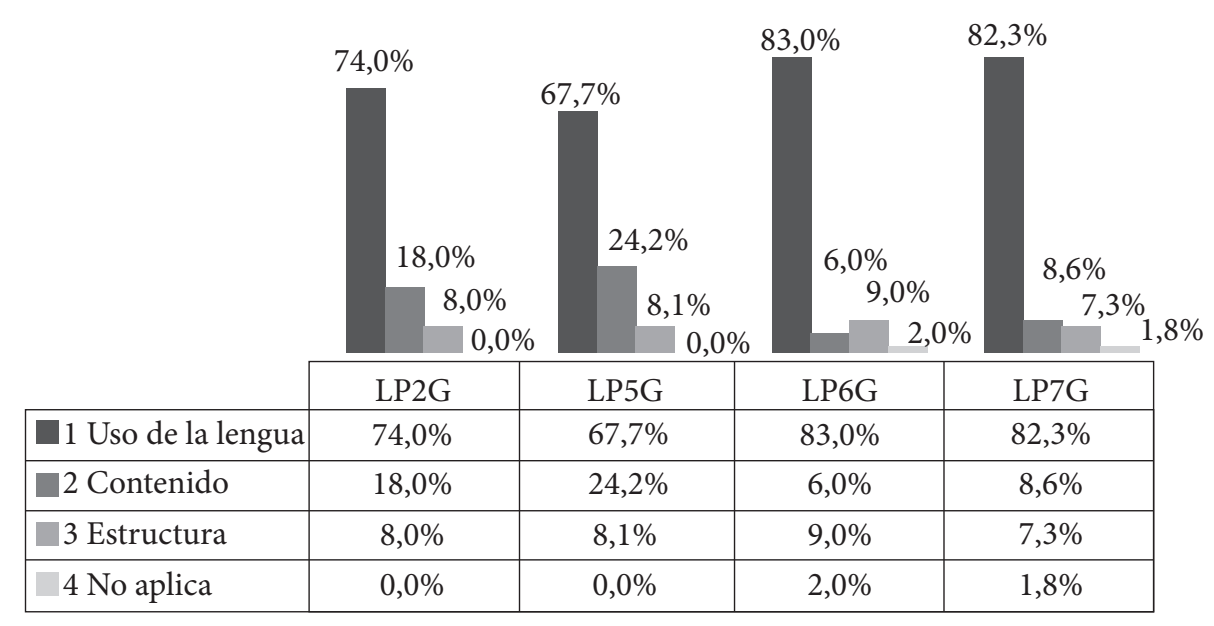

Gráfico 2. Distribución de los CE según la categoría Objeto

$\mathrm{Al}$ revisar el gráfico 2, se advierte un comportamiento poco homogéneo entre los grupos con relación a la categoría Uso de la lengua, en la cual el porcentaje más bajo se ubica en el grupo 2 (67\%) y el más alto en el grupo 6 (83\%). En cambio, en los aspectos de contenido y estructura los resultados son más uniformes. La distribución de los grupos muestra que el mayor número de $\mathrm{CE}$ se asocia al criterio uso de la lengua (79\%), como se aprecia en el gráfico 1 . Tal hecho quiere decir que el mayor número de CE está relacionado con los aspectos del código de la lengua, como léxico, gramática, ortotipografía y puntuación. En este sentido, nuestros resultados coinciden con los de Ferris (1997), quien en su estudio diferenció entre CE marginales y CE finales, cuyos hallazgos revelan que los comentarios marginales son más cortos, mientras que los finales aluden a aspectos semánticos del texto. En cambio, nuestros resultados son mayores que los señalados por Straub y Lunsford (1995), quienes encontraron que el $54 \%$ de los CE estaban asociados a fallos gramaticales y de puntuación. En segundo lugar, dentro de la clasificación de objeto (11\%) encontramos la subcategoría Contenido, que se refiere al nivel de las ideas o los aspectos semánticos del texto. En tercer lugar, ubicamos el aspecto de estructura (8\%); estas correcciones hacen referencia a la organización lógica de la información en el texto. El 1\% de los resultados corresponde al criterio No aplica, es decir, a CE referidos al tono, el registro y la formalidad en el uso de la lengua.

Se presenta a continuación el gráfico 3, con la información que alude a la distribución de los CE según la categoría correctivo.

Al examinar el gráfico 3, se observa que la mayoría de los CE provistos por el docente son de tipo correctivo indirecto y directo, con un $42 \%$ y $37 \%$ respectivamente. Tales datos revelan que la retroalimentación entregada por el docente se ocupa principalmente de entregar pistas o explicaciones con relación a los errores cometidos por los estudiantes, sin proveer la forma correcta. También se advierte una tendencia progresiva en la provisión de retroalimentación correctiva directa en relación con la línea de tiempo, es decir, a medida que avanza en su proceso de formación. En este sentido, el grupo 2 presenta un $12 \%$ de retroalimentación correctiva directa, y el grupo 7 , un $49 \%$, lo cual demuestra tal 
progresión. En cuanto a la retroalimentación correctiva indirecta, se observa un fenómeno opuesto, ya que esta disminuye conforme los estudiantes avanzan en su formación. De esta manera se percibe que el grupo 2 presenta un $64 \%$ de retroalimentación correctiva indirecta, mientras que en el grupo 7 es de solo 32\% en promedio. Los resultados de nuestra investigación son similares a los de Hyland y Hyland (2010), quienes hallaron que el $80 \%$ de los CE provistos por los profesores eran de tipo correctivo. Aunque en nuestra investigación los porcentajes
¿Cómo se orienta la apropiación del género lesson plan mediante la provisión de comentarios escritos en la práctica pedagógica en un programa de formación de profesores de inglés en Chile?

Marta Patricia Meza García, Mónica Tapia Ladino

hallados con relación a la retroalimentación correctiva directa, en particular, son menores, destaca la similitud en el tipo de retroalimentación de errores provista por los profesores, quienes corregían de manera detallada.

En el gráfico 4 se muestran los resultados obtenidos en la categoría Modo. En esta categoría se consideraron los criterios de: felicitación, orden, consejo, requerimiento indirecto, pregunta problema y evaluación negativa.

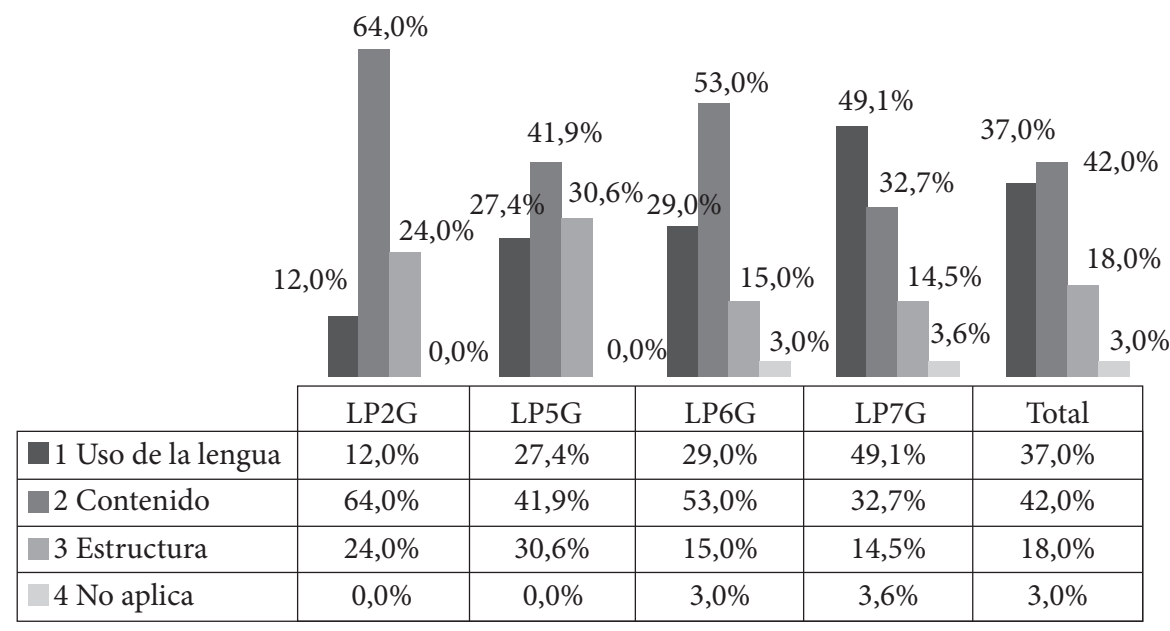

Gráfico 3. Distribución de los CE según la categoría Correctivo

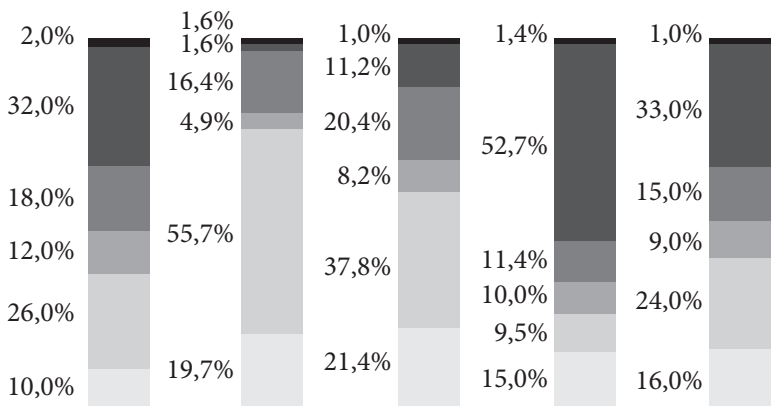

\begin{tabular}{|c|c|c|c|c|c|c|}
\hline & & LP2G & LP5G & LP6G & LP7G & TOTAL \\
\hline ם & 1 Felicitación & $2,0 \%$ & $1,6 \%$ & $1,0 \%$ & $1,4 \%$ & $1,0 \%$ \\
\hline$\square$ & 2 Orden & $32,0 \%$ & $1,6 \%$ & $11,2 \%$ & $52,7 \%$ & $33,0 \%$ \\
\hline $\mathbf{\square}$ & 3 Consejo & $18,0 \%$ & $16,4 \%$ & $20,4 \%$ & $11,4 \%$ & $15,0 \%$ \\
\hline$\square$ & 4 Requerimiento & $12,0 \%$ & $4,9 \%$ & $8,2 \%$ & $10,0 \%$ & $9,0 \%$ \\
\hline$\square$ & 5 Pregunta Problema & $26,0 \%$ & $55,7 \%$ & $37,8 \%$ & $9,5 \%$ & $24,0 \%$ \\
\hline & 6 Evaluación. Negativa & $10,0 \%$ & $19,7 \%$ & $21,4 \%$ & $15,0 \%$ & $16,0 \%$ \\
\hline
\end{tabular}

Gráfico 4. Distribución de los CE según la categoría Modo 
Universidad Pedagógica Nacional

Facultad de Humanidades

Como se advierte en el gráfico 4, la mayoría de los CE se expresan como órdenes (33\%), lo que indica que el profesor solicita de manera directa y explícita los cambios o correcciones que el estudiante debe hacer en el texto. En esta subcategoría el grupo con mayor porcentaje corresponde a la práctica 7 , que alcanza un 52,7 \%, y el más bajo lo encontramos en el grupo 5 (1,6\%). Tales resultados son mayores a los encontrados en el estudio de Straub y Lunsford (1995), quienes hallaron solo un 5\% de este tipo de comentarios en sus datos. Asimismo, encontramos que el modo menos utilizado por nuestro docente es la felicitación (1\%). Nuestros resultados en esta categoría son mucho menores que los hallazgos der la investigación realizada por Straub y Lunsford (1995), quienes encontraron un 9\% de felicitaciones o elogios en su corpus de CE.

En relación con la categoría Evaluación negativa, los resultados muestran una tendencia progresiva en los grupos 2, 5 y 6 , que inician con un $10 \%$ en el primero de los grupos señalados y terminan con un $21 \%$ en el grupo 6 . Sin embargo, el grupo 7 presenta un comportamiento atípico con respecto a la tendencia señalada, con un $15 \%$. En comparación con Straub y Lunsford (1995), nuestros resultados son mayores con un $16 \%$, mientras que el porcentaje encontrado por los autores fue un $7 \%$. En las subcategorías que corresponden a consejo y requerimiento indirecto, los porcentajes fueron del $15 \%$ y $9 \%$ respectivamente. En cuanto a los resultados en la categoría Consejo, nuestros datos (15\%) son mayores con los encontrados por Straub y Lunsford (1995), quienes obtuvieron en su estudio un $9 \%$. Por último, en lo que concierne a la categoría Pregunta-problema los resultados se distribuyen de una manera poco uniforme. No se advierte ninguna tendencia de aumento o disminución, tampoco similitudes entre los resultados de un grupo u otro.

\section{Discusión}

Determinar y describir las características de los CE no es una labor sencilla dado que su función puede variar de acuerdo a los factores sociales y del contexto pedagógico. Nuestra investigación resultó bastante útil en la medida en que nos permitió establecer los rasgos comunes existentes en un conjunto de CE, y determinar al mismo tiempo cómo éstos varían de un semestre a otro. También pudimos establecer algunos rasgos característicos y conductas reiteradas del docente en el momento de proveer retroalimentación escrita. En este sentido, nuestros datos revelan que el profesor realiza sugerencias mayormente en tono imperativo, $y$ hace sugerencias y recomendaciones que ayudan a los estudiantes a mejorar sus competencias escriturales. Desde esta perspectiva, la retroalimentación escrita del docente está orientada no solo al logro del dominio del código en sí mismo, sino además a su dominio con miras al desarrollo de una identidad profesional y su uso en un ámbito pedagógico específico.

En el caso estudiado, el académico ofrece retroalimentación escrita de manera sistemática y natural. El docente mantiene un comportamiento consistente en el tiempo con relación no solo al número de comentarios escritos provistos, sino además a las características de los mismos. Los datos del presente estudio también reflejan algunas similitudes con las investigaciones de Straub y Lundsford (1995) en cuanto al uso del modo imperativo. Los autores señalan que cuando un profesor ofrece comentarios predominantemente correctivos adopta un rol de corrector de pruebas, mientras que un docente que ofrece comentarios imperativos (orden en nuestro caso), cumple el papel de un editor (Straub \& Lundsford, 1995). En nuestro estudio, el profesor privilegia el modo imperativo, se advierte poca presencia de estímulos o felicitaciones en sus comentarios, por lo tanto tiene mayormente el papel de editor. En este sentido, encontramos algunas divergencias con el estudio realizado por Smith (1997). Este autor encuentra un gran porcentaje de comentarios positivos (86\%); fenómeno opuesto a nuestro caso en donde el porcentaje de felicitaciones es de $1 \%$. Una posible razón para explicar este fenómeno es que, debido a las características específicas del género lesson plan, los CE expresados en modo de orden resulten más pertinentes para lograr mayor receptividad en los estudiantes y que los tengan en cuenta. Otra posible 
causa podría ser que en el programa de formación de profesores de Chile se ofrece poca instrucción en la provisión sistemática de retroalimentación escrita. Sin embargo, debido al pequeño corpus analizado en nuestra investigación, no es posible concluir que el comportamiento de este docente responda a una manera generalizada de proveer retroalimentación escrita. Nuestro análisis se hizo sobre material natural y ecológico, es decir, no se instruyó al docente sobre cómo proveer $\mathrm{CE}$, los textos provistos fueron examinados sin intervenirlos en ningún sentido y respetando sus características originales. Dado que la provisión de retroalimentación es una práctica rutinaria, es conveniente capacitar a los profesores para orientar una escritura más autónoma con un profesor que tienda a ser más mentor y menos editor.

Finalmente, en el contexto de la investigación de la retroalimentación escrita, es relevante saber qué decisiones toma el estudiante basado en los comentarios escritos del profesor. Los estudios de la escritura académica en inglés señalan que algunos tipos de retroalimentación promueven diferentes procesos cognitivos (Duijnhouwer, 2010). Desde el punto de vista social, la provisión de CE durante el proceso formativo de los futuros profesores es un recurso que favorece la adopción progresiva de los géneros profesionales; permite ir ajustando los géneros discursivos escritos a los requerimientos de las comunidades donde circularán.

En futuros estudios nos gustaría investigar lo que los estudiantes hacen con los comentarios provistos por su profesor, qué tipo de comentarios consideran más útiles para mejorar sus habilidades en los procesos de escritura y cómo estos CE ayudan al aprendiz en su desarrollo como futuro profesional de la educación. También sería interesante establecer comparaciones en la retroalimentación de varios docentes, ya que en nuestro estudio se realizó bajo un estudio de caso, lo cual no permite sacar conclusiones generales con relación a las características de la retroalimentación provista por el docente. Los estudios sobre comentarios escritos constituyen una disciplina de estudio que necesita más atención, ya que es un campo que entrega abundante informa- ción sobre el lenguaje, la escritura, los procesos de enseñanza-aprendizaje de primera y segunda lengua y el rol social de la escritura, entre otros, por consiguiente se hace necesario seguir investigando sobre los diversos aspectos que ello involucra.

\section{Conclusiones}

El análisis de los CE provistos durante la formación de profesores de inglés como segunda lengua nos permite concluir que son predominantemente simples, correctivos indirectos, sin estrategia, focalizados, expresados como órdenes y prácticamente con ausencia de comentarios positivos. Durante el proceso formativo, los comentarios varían en cuanto a la provisión de estrategia: más al inicio, menos hacia el final y una fuerte atención a solicitar precisión en el uso del código. Los tipos de CE identificados revelan una alta valoración de los docentes sobre el uso de la lengua como elemento característico del género lesson plan, como un recurso que expresa el dominio del inglés como segunda lengua. Dado que la provisión de retroalimentación es una práctica habitual, pensamos que es necesario capacitar a los profesores para orientar una escritura más autónoma con un profesor que tienda a ser más mentor y menos editor.

\section{Referencias bibliográficas}

Bajtin, M. (1985). Estética de la creación verbal. México: Siglo XXI.

Bawarshi, A. \& Reiff, M. (2010): Genre. An introduction to history, theory, research, and pedagogy. Indiana: Parlor Press.

Bazerman, C. (2009. Handbook of research on writing. History, society, school, individual, text. Nueva York: Lawrence Erlbaum Associates.

Bazerman, C., (2010). Genre in a changing world. Nueva York: Routledge.

Bitchener, J. \& Ferris, D. (2012). Written corrective feedback in second language acquisition and writing. New York and London: Routledge Taylor and Francis Group.

Brown, H. D. (2001). Teaching by principles: An interactive approach to language pedagogy. Englewood Cliffs, NJ: Longman. 
Universidad Pedagógica Nacional

Facultad de Humanidades

Duijnhouwer, H. (2010). Feedback effects on students' writing motivation, process and performance. Netherlands: Dutch Interuniversity Centre for Educational Research.

Ellis, R. (2009). A typology of written corrective feedback types. ELT Journal, 63 (2), 97-107.

Ferris, D. (1997). The influence of teacher commentary on student revision. Tesol Quarterly, 31 (2), 315-339.

Ferris, D. (2006). Does error feedback help student writers? New evidences on the short and long-term effects on written error correction. En K. Hyland \& F. Hyland (eds.), Feedback in second language writing: Context and issues (pp. 81- 104). Cambridge: Cambridge University Press.

Ferris, D. (2010). Second language writing research and writing. Corrective feedback in SLA: intersections and practical applications. Studies in Second Language Acquisition, 32, 181- 201.

Harmer, J. (2001). The practice of English Language Teaching. $3^{\text {rd }}$. Essex. ed. Longman.

Haswell, R. (2006). Teaching of Writing in Higher Education. En C. Bazerman, (ed.). Handbook of research on writing: History, society, school, individual, text (pp. 331-346) . Nueva York: Erlbaum.

Hattie, J. \& Timperley, H. (2007). The Power of Feedback. American Educational Research Association. Review of Educational Research, 77 (1), 81-112.

Hernández Sampieri, R. et ál. (2008). Metodología de la investigación. 5. ed., México. Mc Graw Hill.
Hyland, K. (2003). Second language writing. Nueva York: Cambridge University Press.

Hyland, K. \& Hyland, F. (2010). Feedback in second language writing: Context and issues. Nueva York: Cambridge University Press.

Perpigan, H. (2003). Exploring the written feedback dialogue: a research, learning and teaching practice". Language Teaching Research. 7 (2), 259-278.

Smith, S. (1997). The genre of the end comment: Conventions in teacher responses to student writing. College Composition and Communication, 48 (2), 249-268.

Sommers, N. (1982). Responding to student writing. College Composition and Communication, 33 (2), 148-156.

Straub, R. (1997). Students' reactions to teacher comments: An exploratory study. National Council of Teachers of English, 31 (1), 91-119.

Straub, R. \& Lunsford, R. (1995). Twelve readers reading. Responding to college student writing. Cresskill, New Jersey: Hampton Press.

Tapia, M., Arancibia, B. \& Correa, R. (2014b). Los comentarios escritos y su relación con la elaboración de las tesis de pregrado en carreras de pedagogía de la ciudad de Concepción: análisis retórico discursivo de la construcción de un género académico a partir de su género primario. Proyecto Fondo Nacional de Desarrollo Científico y Tecnológico (Fondecyt 1140360). 